\title{
Article \\ Dynamics of Serum Retinol and Alpha-Tocopherol Levels According to Non-Alcoholic Fatty Liver Disease Status
}

\author{
Dongsub Jeon ${ }^{1,+}\left(\mathbb{D}\right.$, Minkook Son ${ }^{2,+}\left(\mathbb{D}\right.$ and Juhyun Shim ${ }^{1, *}$ \\ 1 Liver Center, Asan Medical Center, Department of Gastroenterology, University of Ulsan College of Medicine, \\ Seoul 05505, Korea; thinkdoc87@hanmail.net \\ 2 Department of Biomedical Science and Engineering, Gwangju Institute of Science and Technology, \\ Gwangju 61005, Korea; minkook87@naver.com \\ * Correspondence: s5854@amc.seoul.kr; Tel.: +82-2-3010-5677 \\ + These authors contributed equally to this work.
}

check for updates

Citation: Jeon, D.; Son, M.; Shim, J. Dynamics of Serum Retinol and Alpha-Tocopherol Levels According to Non-Alcoholic Fatty Liver Disease Status. Nutrients 2021, 13, 1720. https://doi.org/10.3390/nu13051720

Academic Editors: Maria Perticone and Angela Sciacqua

Received: 9 April 2021

Accepted: 17 May 2021

Published: 19 May 2021

Publisher's Note: MDPI stays neutra with regard to jurisdictional claims in published maps and institutional affiliations.

Copyright: (c) 2021 by the authors. Licensee MDPI, Basel, Switzerland. This article is an open access article distributed under the terms and conditions of the Creative Commons Attribution (CC BY) license (https:// creativecommons.org/licenses/by/ $4.0 /)$.

\begin{abstract}
The available data on the association between micronutrients in the blood and non-alcoholic fatty liver disease (NAFLD) are limited. To investigate the clinical implications of this relationship, we sought to identify the difference in the serum levels of vitamins A and E according to NAFLD status using data from the seventh Korea National Health and Nutrition Examination Survey. In this cross-sectional study of the Korean population, NAFLD and its severity were defined using prediction models. Differences in the prevalence and severity of NAFLD were analyzed according to serum retinol (vitamin A) and alpha ( $\alpha$ )-tocopherol (vitamin E) levels. Serum levels of retinol and $\alpha$ tocopherol were positively correlated with the prevalence of NAFLD. In most prediction models of the NAFLD subjects, serum retinol deficiency was significantly correlated with advanced fibrosis, while serum $\alpha$-tocopherol levels did not differ between individuals with or without advanced fibrosis. Similar trends were also noted with cholesterol-adjusted levels of $\alpha$-tocopherol. In summary, while circulating concentrations of retinol and $\alpha$-tocopherol were positively associated with the presence of NAFLD, advanced liver fibrosis was only correlated with serum retinol levels. Our findings could provide insight into NAFLD patient care at a micronutrient level.
\end{abstract}

Keywords: non-alcoholic fatty liver disease; vitamin A; vitamin E; advanced liver fibrosis

\section{Introduction}

Non-alcoholic fatty liver disease (NAFLD) is the most common liver-related disorder globally. It is currently present in approximately $25 \%$ of the world's population, and this figure continues to rise due to the increased prevalence of obesity and the aging population [1]. NAFLD varies from simple hepatic steatosis without inflammation or fibrosis to non-alcoholic steatohepatitis (NASH), which can lead to cirrhosis and hepatocellular carcinoma [2]. Therefore, effectively preventing and treating NAFLD has become increasingly necessary due to the clinical and economic burden of the disease. To do this, diagnosing NAFLD early is crucial. To meet the practical need for noninvasive but accurate methods to diagnose NAFLD and predict the risk of NASH, numerous studies have investigated prediction models using single or combined biochemical and/or anthropometric parameters [3-8]. Various hepatic steatosis formulae for the diagnosis of NAFLD have proven comparable to standard ultrasonography in multiple cohorts [5,9-11], and a recent metaanalysis also reported acceptable performance of the simple biological scoring systems, including the BMI-AST / ALT Ratio-Diabetes (BARD) score and the Fibrosis-4 (FIB-4) index, when compared to transient elastography in the detection of advanced fibrosis [12-15].

Among the known factors associated with NAFLD, the pathogenic role of macronutrients is well-established in NAFLD. However, the contribution of micronutrients to NAFLD pathogenesis has garnered less attention than to obesity [16]. Vitamins are essential micronutrients that regulate cellular growth and metabolism and are categorized as either 
fat-soluble or water-soluble. NAFLD may disturb the metabolism of fat-soluble vitamins (A, D, E, and K) [17]. Retinoic acid, an active metabolite of vitamin A, is associated with the pathogenesis of NAFLD through its effect on hepatic lipid metabolism and insulin resistance [18]. Vitamin E, another fat-soluble vitamin, has antioxidant, anti-inflammatory, and antifibrotic effects [19].

Interestingly, findings on serum levels of vitamin A and E in NAFLD are controversial. According to Mazidi et al.'s cross-sectional study using the Nutrition and Health Examination Surveys data, serum vitamin A and E levels are negatively associated with NAFLD severity [20]. On the other hand, in a cross-sectional study conducted by Pettinelli et al., serum retinol levels were higher in NAFLD patients than in healthy liver donors without NAFLD [21]. Additionally, serum alpha $(\alpha)$-tocopherol levels were positively associated with central adiposity after adjusting for body fat in another cross-sectional study [22].

Recently, a study concluded that high serum retinol and $\alpha$-tocopherol levels were associated with an increased risk of metabolic syndrome in South Korean patients [23]. Given that NAFLD is a hepatic manifestation of metabolic syndrome, we hypothesized positive correlations between the vitamin concentrations and NAFLD. Since vitamin A is metabolized and maintains its homeostasis in the liver, NAFLD could have a more direct influence on vitamin A homeostasis than metabolic syndrome has [18]. Additionally, since vitamin $\mathrm{E}$ is used as a treatment for NAFLD accompanied by NASH, monitoring and maintaining vitamin E status in NAFLD patients is essential [24].

Therefore, we decided to conduct a large population-based study using public data on 4448 subjects from South Korea to determine the association of serum retinol and $\alpha$-tocopherol levels according to disease status in subjects with NAFLD diagnosed by noninvasive laboratory methods and to provide an informative reference for the nutritional and clinical management of patients.

\section{Materials and Methods}

\subsection{Data Information and Study Population}

The KNHANES (Korea National Health and Nutrition Examination Survey) is a cross-sectional survey and a nationally representative database of Korea managed by the Korea Centers for Disease Control and Prevention (KCDCP) [25]. This study used data from the seventh KNHANES VII (2016-2018) [26]. The KNHANES database includes the results of physical examinations, laboratory tests, health-related interviews, and nutritional surveys. Informed consent was acquired from subjects for their voluntary participation, and the KNHANES has been approved by the Institutional Review Board of the KCDCP. This study's protocol was reviewed and approved by the Institutional Review Board of Asan Medical Center (IRB number: 2020-1893), which waived the need for informed consent because the data were anonymized and analyzed retrospectively. This study was performed in accordance with the Declaration of Helsinki.

From the total 24,269 subjects retrieved from the KNHANES VII database, we excluded 17,079 subjects without serum retinol and $\alpha$-tocopherol levels. We excluded those aged $<20$ years $(n=825)$, those with a previous history of any cancer $(n=111)$, those who were pregnant $(n=20)$, and those with missing variables $(n=861)$. Additionally, subjects who met the following criteria were excluded from the initial analysis: (a) those with positive markers for hepatitis B virus (HBV) or hepatitis C virus $(\mathrm{HCV})(n=197)$, (b) severe alcoholics with daily drinking $(n=720)$, and (c) those with liver cirrhosis $(n=8)$. This excluded population $(n=925)$ was later included in the study population for a sensitivity analysis. Finally, this study comprised 4448 subjects (1677 males and 2771 females). The study population was divided into quartile groups according to serum retinol and $\alpha$ tocopherol levels. Subsequently, using fatty liver prediction models, the patients with NAFLD were further divided into quartile groups according to serum retinol and $\alpha$ tocopherol levels. A flow diagram for this study is presented in Figure 1. 


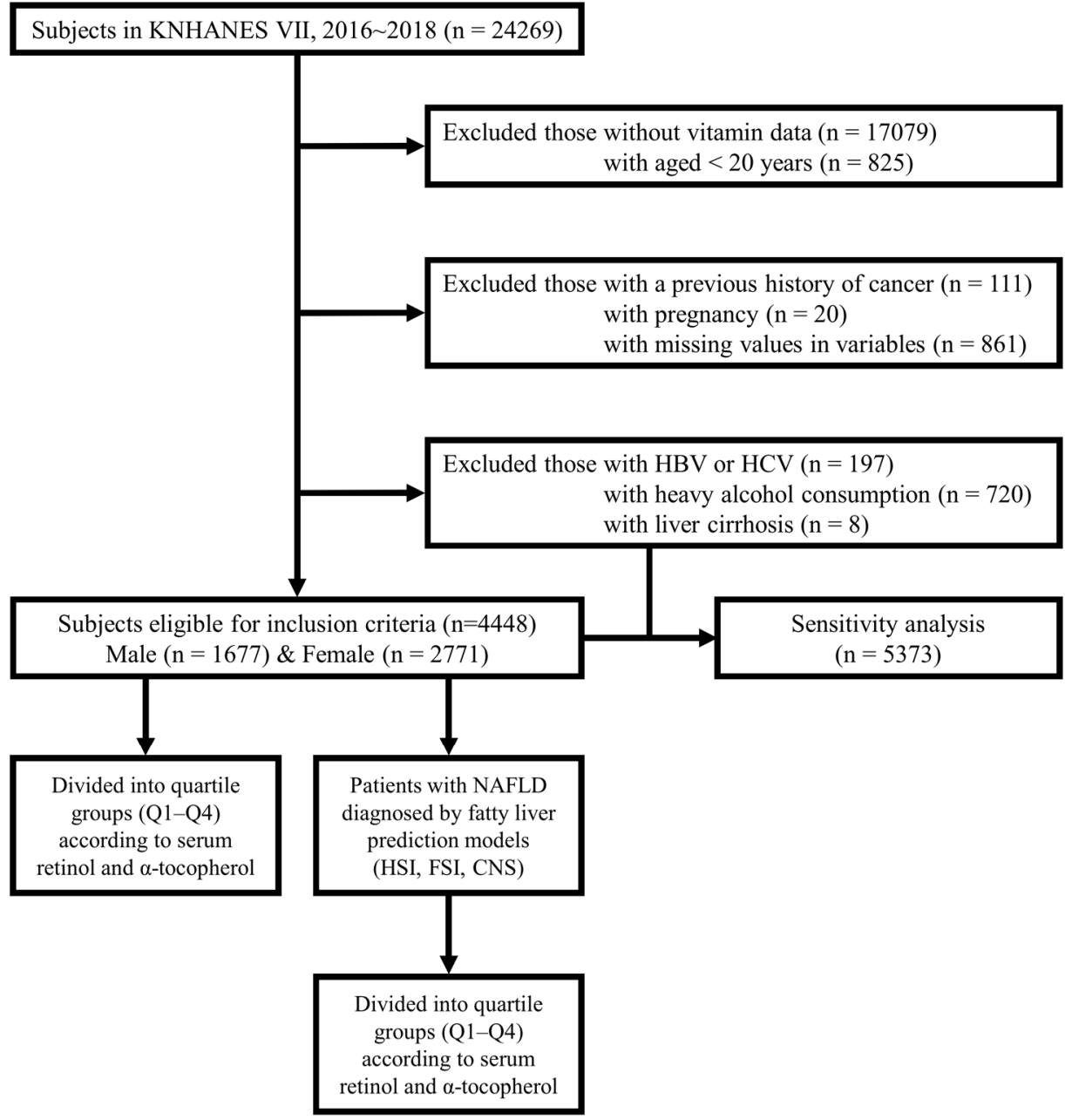

Figure 1. Flow diagram of study population. From the total 24,269 subjects retrieved from the KNHANES VII database, we excluded the following subjects: (1) 17,079 subjects without data on serum retinol and alpha $(\alpha)$-tocopherol levels; (2) 825 aged < 20 years; (3) 111 with a previous history of any cancer; (4) 20 who were pregnant; and (5) 861 with missing variables. An additional 926 subjects, (1) 197 positive for HBV or HCV; (2) 720 heavy drinkers; and (3) 8 with liver cirrhosis were also excluded from the initial analysis but included in the later sensitivity analysis. Finally, 4448 subjects ( 1677 males and 2771 females) were included. All included individuals were divided into quartile groups according to serum retinol and $\alpha$-tocopherol levels. KNHANES VII, 7 th Korea National Health And Nutrition Examination Survey; HBV, hepatitis B virus; HCV, hepatitis C virus; Q, quartile; NAFLD, non-alcoholic fatty liver disease; HSI, hepatic steatosis index; FSI, Framingham steatosis index; CNS, comprehensive NAFLD score.

\subsection{Clinical Variable Measurements and Laboratory Analyses}

Anthropometric measurements were performed by trained staff. The body mass index (BMI) was calculated using the formula weight $(\mathrm{kg})$ divided by height squared $\left(\mathrm{m}^{2}\right)$. Subjects were categorized as obese if their BMI was $\geq 25 \mathrm{~kg} / \mathrm{m}^{2}$ and as non-obese if the BMI was $<25 \mathrm{~kg} / \mathrm{m}^{2}$ [27].

All subjects fasted for at least $8 \mathrm{~h}$ prior to taking blood samples. The blood samples were refrigerated immediately for transportation to the Central Testing Institute (NeoDin Medical Institute, Seoul, Korea). Fasting glucose, total cholesterol, triglycerides, highdensity lipoprotein cholesterol (HDL-C), and creatinine were measured by the hexokinase UV, enzymatic, homogeneous enzymatic colorimetric, Jaffe rate-blanked, and compensated methods, respectively, using the Hitachi Automatic Analyzer 7600-210 (Hitachi, Tokyo, Japan). For the quantification of serum retinol and $\alpha$-tocopherol levels, high-performance 
liquid chromatography-fluorescence with Agilent 1200 (Agilent, Santa Clara, CA, USA) was applied using Chromsystems (Chromsystems Instruments \& Chemicals, Munich, Germany). The normal reference range for serum retinol levels in adults is $0.30-0.70 \mathrm{mg} / \mathrm{L}$, and for serum $\alpha$-tocopherol levels in adults, it is $5.00-20.00 \mathrm{mg} / \mathrm{L}$. Further information on the blood tests is described on the KCDCP website [25]. The estimated glomerular filtration rate (eGFR) was determined from the Modification of Diet in Renal Disease equation [28].

Hypertensive status was divided into three categories: (a) hypertension was defined as systolic blood pressure (SBP) $\geq 140 \mathrm{mmHg}$ or diastolic blood pressure (DBP) $\geq 90 \mathrm{mmHg}$ or taking anti-hypertensive medication; (b) pre-hypertension was defined as $120 \mathrm{mmHg} \leq$ $\mathrm{SBP}<140 \mathrm{mmHg}$ or $80 \mathrm{mmHg} \leq \mathrm{DBP}<90 \mathrm{mmHg}$; and (c) normal was defined as $\mathrm{SBP}<$ $120 \mathrm{mmHg}$ and DBP $<80 \mathrm{mmHg}$ [29]. Diabetic status was divided into three groups: (a) diabetes was defined as fasting blood glucose $(\mathrm{FBG}) \geq 126 \mathrm{mg} / \mathrm{dL}$ or taking anti-diabetic medication; (b) pre-diabetes was defined as $100 \mathrm{mg} / \mathrm{dL} \leq \mathrm{FBG}<126 \mathrm{mg} / \mathrm{dL}$; and (c) normal was defined as FBG $<100 \mathrm{mg} / \mathrm{dL}$ [30]. Dyslipidemia status was categorized into two groups using the dyslipidemia criteria for Koreans, with cutoff values as follows: total cholesterol $\geq 240 \mathrm{mg} / \mathrm{dL}$, triglycerides $\geq 200 \mathrm{mg} / \mathrm{dL}$, or HDL-C $\leq 40 \mathrm{mg} / \mathrm{dL}$ [31].

Demographic and medical information was obtained from self-report questionnaires and face-to-face interviews with trained staff. Income level was classified into four groups. Smoking status was categorized into current smokers, ex-smokers, and non-smokers. Alcohol consumption status was categorized into two groups: (a) non-consumers, with no alcohol intake in the past year or less than once a month, and (b) alcohol consumers, with alcohol intake more than once a month. Physical exercise status was categorized into two groups: (a) the regular exercise group, with moderately intense exercise at least $150 \mathrm{~min}$ within a week, intense exercise at least $75 \mathrm{~min}$ within a week, or mixed exercise equivalent to the above level ( $1 \mathrm{~min}$ of intense exercise equivalent to $2 \mathrm{~min}$ of moderately intense exercise), and (b) the non-regular exercise group, with physical activity less than the level described above [32].

Dietary information was investigated with the 24-hour recall method. According to the Dietary Reference Intakes for Koreans criteria, vitamin A was measured using retinol equivalents (RE) and derived from the following equation: RE $(\mu \mathrm{g})=$ retinol $(\mu \mathrm{g})+\beta-$ carotenes $/ 6(\mu \mathrm{g})$ [33]. However, information on vitamin E consumption was not included in the KNHANES VII database. Use of vitamin supplements was categorized into nonsupplement users (no vitamin supplements) and supplement users (vitamin supplements for more than two weeks within a 1-year period).

\subsection{Definitions of Hepatic Steatosis and Advanced Fibrosis}

NAFLD was defined using the following fatty liver prediction models: (a) the hepatic steatosis index (HSI), (b) the Framingham steatosis index (FSI), and (c) the comprehensive NAFLD score (CNS) $[4,5,34]$. The BARD and FIB-4 scores were applied to define advanced fibrosis and were calculated in subjects classified as having NAFLD using the aforementioned prediction models $[14,15]$. All prediction models are described in Table S1.

\subsection{Statistical Analysis}

The subjects' characteristics were described according to vitamin (serum retinol and $\alpha$-tocopherol) and NAFLD (HSI, FSI, and CNS) status. Continuous variables are reported as mean with standard deviation, and categorical variables are reported as number with percentage. Characteristics of the quartile groups for serum retinol and $\alpha$-tocopherol were compared using the one-way analysis of variance and the chi-square test. The association between NAFLD status and vitamin (serum retinol and $\alpha$-tocopherol) levels was estimated using logistic regression models. The model for serum retinol was adjusted for age, sex, BMI, GFR, hypertension, diabetes, dyslipidemia status, income level, smoking status, alcohol consumption, exercise status, use of vitamin supplements, and daily dietary intake of vitamin A. For the model for $\alpha$-tocopherol, all the aforementioned variables except the daily dietary intake of vitamin A, were included for adjustment. Subgroup 
analyses were performed according to the presence of obesity and diabetes. The association between advanced fibrosis (BARD and FIB-4) status and vitamin (serum retinol and $\alpha$ tocopherol) levels was estimated using the same multivariable-adjusted logistic regression models. Moreover, we performed an additional analysis with $\alpha$-tocopherol adjusted for cholesterol ( $\alpha$-tocopherol/cholesterol ratio) because $\alpha$-tocopherol can be affected by lipid metabolism [35]. Data collection and statistical analyses were performed with SPSS software version 20 (IBM, SPSS Inc., Chicago, IL, USA). The $p$-value $<0.05$ was considered statistically significant.

\section{Results}

\subsection{Baseline Characteristics of Study Subjects}

The baseline characteristics of the study subjects are summarized according to the quartile groups for serum retinol and $\alpha$-tocopherol in Tables 1 and 2, respectively. As serum retinol and $\alpha$-tocopherol increased, the mean age, SBP, BMI, FBG, total cholesterol, and triglycerides increased, but the mean HDL-C and GFR decreased. The subjects in the higher quartiles for serum retinol and $\alpha$-tocopherol were more likely to be male; had a greater prevalence of hypertension, diabetes, and dyslipidemia; were more likely to be current smokers and alcohol consumers; and were more likely to use vitamin supplements. There were no significant differences in the proportion who exercised regularly or in the daily dietary intake of vitamin $\mathrm{A}$. The mean values of serum retinol according to quartile from Q1 to Q4 were $0.32 \mathrm{mg} / \mathrm{L}, 0.43 \mathrm{mg} / \mathrm{L}, 0.53 \mathrm{mg} / \mathrm{L}$, and $0.74 \mathrm{mg} / \mathrm{L}$, respectively, while the mean values of $\alpha$-tocopherol were $8.7 \mathrm{mg} / \mathrm{L}, 11.4 \mathrm{mg} / \mathrm{L}, 13.9 \mathrm{mg} / \mathrm{L}$, and $20.3 \mathrm{mg} / \mathrm{L}$, respectively. As the quartile of serum retinol and $\alpha$-tocopherol increased, the prevalence of NAFLD assessed according to the HSI, FSI, and CNS also increased. The proportions of patients with serum retinol and $\alpha$-tocopherol levels according to quartile using different NAFLD scores by quartile are presented in Figure 2 and in Figures S1 and S2. In addition, the proportion of subjects with serum retinol levels according to the reference value of different NAFLD scores are presented in Figure S3. Serum retinol and $\alpha$-tocopherol had strong positive associations with all NAFLD prediction models ( $p s<0.0001$ for the trends).

Table 1. Characteristics of subjects according to quartile group for serum retinol.

\begin{tabular}{|c|c|c|c|c|c|}
\hline \multirow{2}{*}{$\begin{array}{c}\text { Total } \\
(n=4448)\end{array}$} & \multicolumn{4}{|c|}{ Serum Retinol } & \multirow[b]{2}{*}{$p$-Value } \\
\hline & $\begin{array}{c}\text { Q1 * } \\
(n=1112)\end{array}$ & $\begin{array}{c}\mathrm{Q} 2 * \\
(n=1112)\end{array}$ & $\begin{array}{c}\mathrm{Q} 3 * \\
(n=1112)\end{array}$ & $\begin{array}{c}\mathrm{Q} 4 * \\
(n=1112)\end{array}$ & \\
\hline Age (years) & $45.9 \pm 17.2$ & $49.4 \pm 16.1$ & $51.8 \pm 15.3$ & $54.2 \pm 14.7$ & $<0.001$ \\
\hline Sex (male) & $210(18.9)$ & $356(32.0)$ & $488(43.9)$ & $623(56.0)$ & $<0.001$ \\
\hline Income & & & & & 0.04 \\
\hline 1st quartile (lowest) & $279(25.1)$ & $295(26.5)$ & $266(23.9)$ & $253(22.8)$ & \\
\hline 2nd quartile & $301(27.1)$ & $244(21.9)$ & $269(24.2)$ & $305(27.4)$ & \\
\hline 3rd quartile & $266(23.9)$ & $301(27.1)$ & $304(27.3)$ & $270(24.3)$ & \\
\hline 4th quartile (highest) & $266(23.9)$ & $272(24.5)$ & $273(24.6)$ & $284(25.5)$ & \\
\hline $\mathrm{SBP}(\mathrm{mmHg})$ & $113.1 \pm 16.0$ & $116.8 \pm 16.8$ & $119.3 \pm 16.6$ & $121.8 \pm 16.4$ & $<0.001$ \\
\hline BMI $\left(\mathrm{kg} / \mathrm{m}^{2}\right)$ & $23.1 \pm 3.7$ & $23.6 \pm 3.7$ & $24.0 \pm 3.3$ & $24.4 \pm 3.3$ & $<0.001$ \\
\hline FBG (mg/dL) & $95.4 \pm 19.3$ & $98.8 \pm 22.1$ & $103.1 \pm 28.7$ & $105.0 \pm 27.0$ & $<0.001$ \\
\hline Total cholesterol (mg/dL) & $185.3 \pm 32.6$ & $192.0 \pm 36.1$ & $195.8 \pm 37.4$ & $197.3 \pm 40.1$ & $<0.001$ \\
\hline Triglycerides (mg/dL) & $91.0 \pm 56.7$ & $118.9 \pm 88.8$ & $134.8 \pm 82.9$ & $170.5 \pm 119.9$ & $<0.001$ \\
\hline HDL-C (mg/dL) & $53.4 \pm 12.4$ & $51.6 \pm 12.9$ & $50.7 \pm 12.6$ & $49.5 \pm 12.3$ & $<0.001$ \\
\hline GFR $\left(\mathrm{mL} / \mathrm{min} / 1.73 \mathrm{~m}^{2}\right)$ & $95.6 \pm 18.2$ & $92.3 \pm 17.5$ & $88.5 \pm 16.8$ & $84.1 \pm 23.5$ & $<0.001$ \\
\hline Hypertension & $223(20.1)$ & $291(26.2)$ & $355(31.9)$ & $456(41.0)$ & $<0.001$ \\
\hline Diabetes & $78(7.0)$ & $102(9.2)$ & $154(13.8)$ & $197(17.7)$ & $<0.001$ \\
\hline Dyslipidemia & $282(25.4)$ & $385(34.6)$ & $521(46.9)$ & $634(57.0)$ & $<0.001$ \\
\hline Current smoker & $62(5.6)$ & $107(9.6)$ & 132 (11.9) & $240(21.6)$ & $<0.001$ \\
\hline Alcohol consumption & $322(29.0)$ & $382(34.4)$ & $431(38.8)$ & $541(48.7)$ & $<0.001$ \\
\hline Regular exercise & $480(43.2)$ & $485(43.6)$ & $477(42.9)$ & $453(40.7)$ & 0.53 \\
\hline Use of supplements & $534(48.0 \%)$ & $573(51.5 \%)$ & $601(54.0 \%)$ & $628(56.5 \%)$ & 0.001 \\
\hline Dietary vitamin A intake ( $\mu \mathrm{gRE} /$ day) & $596.3 \pm 677.9$ & $634.6 \pm 603.6$ & $620.7 \pm 536.4$ & $607.1 \pm 493.5$ & 0.81 \\
\hline
\end{tabular}


Table 1. Cont.

\begin{tabular}{|c|c|c|c|c|c|}
\hline \multirow[b]{2}{*}{$\begin{array}{c}\text { Total } \\
(n=4448)\end{array}$} & \multicolumn{4}{|c|}{ Serum Retinol } & \multirow[b]{2}{*}{$p$-Value } \\
\hline & $\begin{array}{c}\mathrm{Q} 1 * \\
(n=1112)\end{array}$ & $\begin{array}{c}\mathrm{Q} 2 * \\
(n=1112)\end{array}$ & $\begin{array}{c}\text { Q3 } \\
(n=1112)\end{array}$ & $\begin{array}{c}\mathrm{Q} 4 * \\
(n=1112)\end{array}$ & \\
\hline Serum retinol level (mg/L) & $0.32 \pm 0.06$ & $0.43 \pm 0.03$ & $0.53 \pm 0.03$ & $0.74 \pm 0.15$ & $<0.001$ \\
\hline$<0.3 \mathrm{mg} / \mathrm{L}$ & $325(29.2)$ & $0(0)$ & $0(0)$ & $0(0)$ & \\
\hline $0.3-0.7 \mathrm{mg} / \mathrm{L}$ & 787 (70.8) & $1112(100)$ & $1112(100)$ & $584(52.5)$ & \\
\hline$>0.7 \mathrm{mg} / \mathrm{L}$ & $0(0)$ & $0(0)$ & $0(0)$ & $528(47.5)$ & \\
\hline NAFLD assessed by HSI & $138(12.4)$ & $193(17.4)$ & $244(21.9)$ & $287(25.8)$ & $<0.001$ \\
\hline NAFLD assessed by FSI & $151(13.6)$ & $227(20.4)$ & $287(25.8)$ & $429(38.6)$ & $<0.001$ \\
\hline NAFLD assessed by CNS & $325(29.2)$ & $461(41.5)$ & $578(52.0)$ & $729(65.6)$ & $<0.001$ \\
\hline
\end{tabular}

Continuous variables are reported as mean with standard deviation, and categorical variables are reported as number with percentage. Serum retinol levels were categorized into quartiles. * Q1, <0.39 mg/L; Q2, 0.39-0.48 mg/L; Q3, 0.48-0.59 mg/L; and Q4, >0.59 mg/L. SBP, systolic blood pressure; BMI, body mass index; HDL-C, high-density lipoprotein cholesterol; GFR, glomerular filtration rate; RE, retinol equivalent; NAFLD, non-alcoholic fatty liver disease; HSI, hepatic steatosis index; FBG, fasting blood glucose; FSI, Framingham steatosis index; CNS, comprehensive NAFLD score. Data are expressed as mean \pm standard deviation or number (\%).

Table 2. Characteristics of subjects according to quartile group for alpha ( $\alpha$ )-tocopherol.

\begin{tabular}{|c|c|c|c|c|c|}
\hline \multirow{2}{*}{$\begin{array}{c}\text { Total } \\
(n=4448)\end{array}$} & \multicolumn{4}{|c|}{$\alpha$-Tocopherol } & \multirow[b]{2}{*}{$p$-Value } \\
\hline & $\begin{array}{c}\mathrm{Q1}{ }^{*} \\
(n=1112)\end{array}$ & $\begin{array}{c}\mathrm{Q} 2{ }^{*} \\
(n=1112)\end{array}$ & $\begin{array}{c}\mathrm{Q} 3{ }^{*} \\
(n=1112)\end{array}$ & $\begin{array}{c}\mathrm{Q} 4 * \\
(n=1112)\end{array}$ & \\
\hline Age (years) & $46.0 \pm 17.7$ & $48.2 \pm 16.3$ & $52.5 \pm 15.1$ & $54.6 \pm 13.7$ & $<0.001$ \\
\hline Sex (male) & $481(43.3)$ & 415 (37.3) & 403 (36.2) & $378(34.0)$ & $<0.001$ \\
\hline Income & & & & & 0.006 \\
\hline 1st quartile (lowest) & $311(28.0)$ & $278(25.0)$ & $277(24.9)$ & $227(20.5)$ & \\
\hline 2nd quartile & $273(24.6)$ & $279(25.1)$ & $269(24.2)$ & $298(26.8)$ & \\
\hline 3rd quartile & $285(25.6)$ & $292(26.2)$ & $271(24.4)$ & $293(26.3)$ & \\
\hline 4th quartile (highest) & $243(21.8)$ & $263(23.7)$ & $295(26.5)$ & $294(26.4)$ & \\
\hline $\mathrm{SBP}(\mathrm{mmHg})$ & $113.9 \pm 15.7$ & $116.0 \pm 16.4$ & $119.6 \pm 17.1$ & $121.5 \pm 16.9$ & $<0.001$ \\
\hline $\mathrm{BMI}\left(\mathrm{kg} / \mathrm{m}^{2}\right)$ & $23.3 \pm 3.7$ & $23.6 \pm 3.5$ & $23.9 \pm 3.4$ & $24.3 \pm 3.4$ & $<0.001$ \\
\hline $\mathrm{FBG}(\mathrm{mg} / \mathrm{dL})$ & $98.8 \pm 24.0$ & $98.0 \pm 19.5$ & $101.5 \pm 27.3$ & $104.1 \pm 27.4$ & $<0.001$ \\
\hline Total cholesterol (mg/dL) & $167.4 \pm 29.9$ & $186.1 \pm 27.9$ & $200.0 \pm 32.4$ & $216.8 \pm 37.6$ & $<0.001$ \\
\hline Triglycerides (mg/dL) & $93.7 \pm 49.6$ & $110.8 \pm 65.3$ & $131.9 \pm 71.1$ & $178.7 \pm 140.7$ & $<0.001$ \\
\hline HDL-C (mg/dL) & $49.7 \pm 10.8$ & $51.8 \pm 12.3$ & $52.1 \pm 13.5$ & $51.5 \pm 13.6$ & 0.001 \\
\hline GFR $\left(\mathrm{mL} / \mathrm{min} / 1.73 \mathrm{~m}^{2}\right)$ & $93.6 \pm 20.1$ & $91.0 \pm 18.4$ & $88.7 \pm 18.1$ & $87.0 \pm 21.3$ & $<0.001$ \\
\hline Hypertension & $279(25.1)$ & $289(26.0)$ & $369(33.2)$ & $388(34.9)$ & $<0.001$ \\
\hline Diabetes & $139(12.5)$ & $100(9.0)$ & $132(11.9)$ & $160(14.4)$ & $<0.001$ \\
\hline Dyslipidemia & $341(30.7)$ & $346(31.1)$ & $481(43.3)$ & $654(58.8)$ & $<0.001$ \\
\hline Current smoker & $167(15.0)$ & $104(9.4)$ & $131(11.8)$ & $139(12.5)$ & 0.003 \\
\hline Alcohol consumption & $407(36.6)$ & $458(41.2)$ & $419(37.7)$ & $392(35.3)$ & 0.03 \\
\hline Regular exercise & $471(42.4)$ & $482(43.3)$ & $449(40.4)$ & $493(44.3)$ & 0.27 \\
\hline Use of supplements & $478(43.0)$ & $530(47.7)$ & $619(55.7)$ & $709(63.8)$ & $<0.001$ \\
\hline$\alpha$-tocopherol level (mg/L) & $8.7 \pm 1.3$ & $11.4 \pm 0.7$ & $13.9 \pm 0.9$ & $20.3 \pm 5.9$ & $<0.001$ \\
\hline$<5 \mathrm{mg} / \mathrm{L}$ & $12(1.1)$ & $0(0)$ & $0(0)$ & $0(0)$ & \\
\hline $5-20 \mathrm{mg} / \mathrm{L}$ & $1100(98.9)$ & $1112(100)$ & $1112(100)$ & $729(65.6)$ & \\
\hline$>20 \mathrm{mg} / \mathrm{L}$ & $0(0)$ & $0(0)$ & $0(0)$ & $383(34.4)$ & \\
\hline NAFLD assessed by HSI & $192(17.3)$ & $184(16.5)$ & $225(20.2)$ & $261(23.5)$ & $<0.001$ \\
\hline NAFLD assessed by FSI & $206(18.5)$ & $205(18.4)$ & $287(25.8)$ & $396(35.6)$ & $<0.001$ \\
\hline NAFLD assessed by CNS & $398(35.8)$ & $451(40.6)$ & $572(51.4)$ & $672(60.4)$ & $<0.001$ \\
\hline
\end{tabular}

Continuous variables are reported as mean with standard deviation, and categorical variables are reported as number with percentage. Serum $\alpha$-tocopherol levels were categorized into quartiles. * Q1, <10.3 mg/L; Q2, 10.3-12.5 mg/L; Q3, 12.5-15.6 mg/L; and Q4, >15.6 mg/L. SBP, systolic blood pressure; BMI, body mass index; HDL-C, high-density lipoprotein cholesterol; GFR, glomerular filtration rate; NAFLD, non-alcoholic fatty liver disease; HSI, hepatic steatosis index; FBG, fasting blood glucose; FSI, Framingham steatosis index; CNS, comprehensive NAFLD score. Data are expressed as mean \pm standard deviation or number $(\%)$. 
(a) All subjects

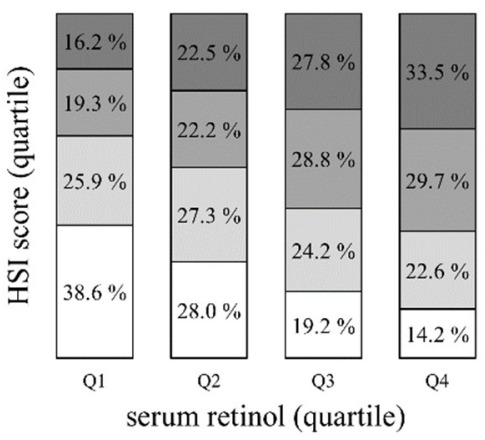

口Q1 पQ2 वQ3 वQ4

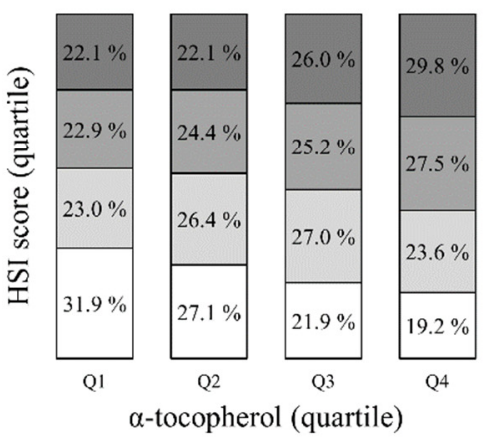

口Q1 वQ2 वQ3 वQ4 (b) Male subjects

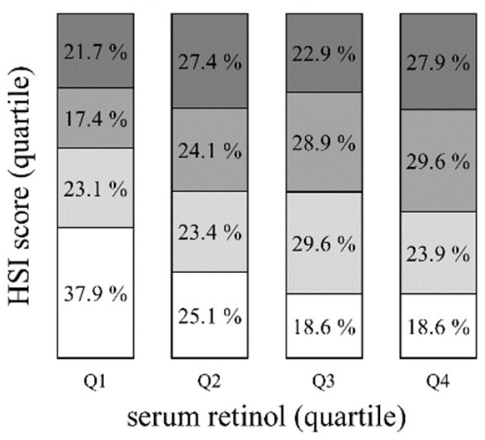

口Q1 वQ2 वQ3 वQ4

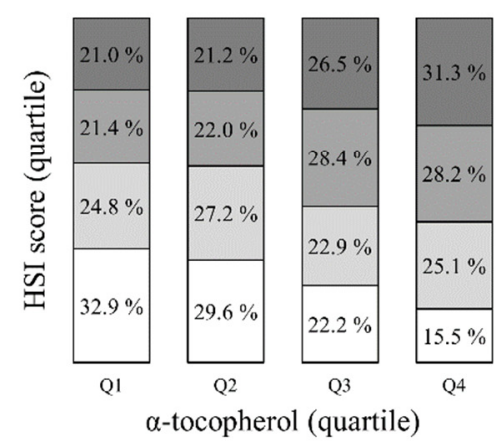

口Q1 वQ2 वQ3 वQ4 (c) Female subjects

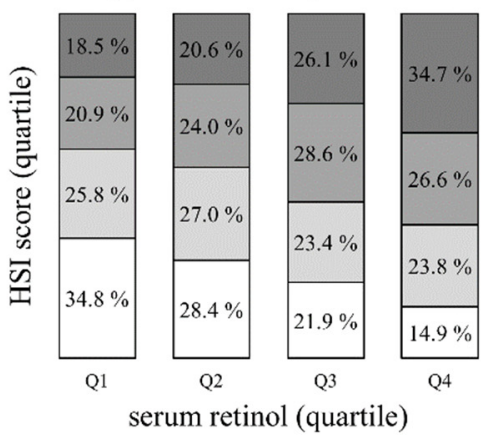

$\square Q 1 \square Q 2 \square Q 3 \square Q 4$

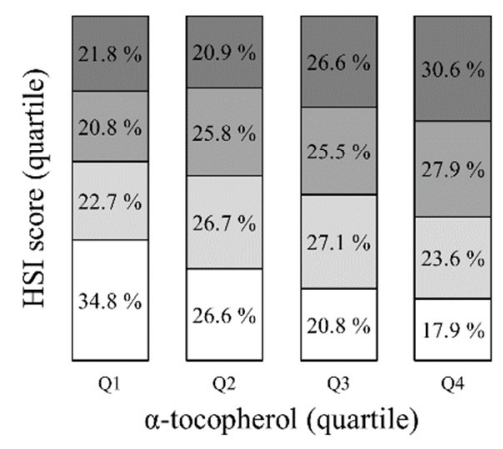

$\square \mathrm{Q} 1 \square \mathrm{Q} 2 \square \mathrm{Q} 3 \square \mathrm{Q} 4$

Figure 2. Proportion of subjects with serum retinol and $\alpha$-tocopherol levels according to quartile with predicted NAFLD prevalence (HSI) by quartile. The study population was divided into quartile groups according to NAFLD (HSI) and vitamin (serum retinol and $\alpha$-tocopherol) levels, respectively. Serum retinol and $\alpha$-tocopherol had strong positive associations with the NAFLD (HSI) prediction model ( $p$ s $<0.0001$ for the trends). NAFLD, non-alcoholic fatty liver disease; HSI, hepatic steatosis index; $Q$, quartile.

\subsection{Association between Vitamins and NAFLD Assessed Using Different Prediction Models (HSI, FSI, CNS)}

The multivariable adjustment was performed for variables that could affect the prevalence of NAFLD. The adjusted odds ratios (AORs) and 95\% confidence intervals (CIs) for the association between the vitamins and NAFLD which was assessed using the different prediction models are shown in Tables 3 and 4. Since there was a disparity for sex, we examined the association between the vitamins and NAFLD after stratification by sex. In all subjects, serum retinol and $\alpha$-tocopherol were significantly associated with NAFLD regardless of the prediction model. Specifically, the AORs and 95\% CIs for NAFLD comparing Q4 with Q1 were 1.67 (1.22-2.45), 2.34 (1.65-3.32), and 3.28 (2.33-4.61) for serum retinol and 1.44 (1.01-2.05), 3.96 (2.83-5.56), and 3.39 (2.44-4.71) for $\alpha$-tocopherol in the HSI, FSI, and CNS models, respectively. In the analysis stratified by sex, there were significant associations between the vitamins and NAFLD for all prediction models, except for HSI in male subjects. 
Table 3. AORs with 95\% CIs for association between serum retinol and NAFLD assessed using different prediction models.

\begin{tabular}{|c|c|c|c|c|c|c|c|c|c|}
\hline \multirow{2}{*}{ Serum Retinol } & \multicolumn{3}{|c|}{ NAFLD Assessed by HSI } & \multicolumn{3}{|c|}{ NAFLD Assessed by FSI } & \multicolumn{3}{|c|}{ NAFLD Assessed by CNS } \\
\hline & AOR * & $95 \%$ CI & $p$-Value & AOR * & $95 \%$ CI & $p$-Value & AOR * & $95 \%$ CI & $p$-Value \\
\hline \multicolumn{10}{|l|}{$\begin{array}{l}\text { All subjects } \\
(n=4448)\end{array}$} \\
\hline Q1 & 1 & & & 1 & & & 1 & & \\
\hline Q2 & 1.47 & $0.99,2.17$ & 0.06 & 1.38 & $0.96,1.98$ & 0.08 & 1.69 & $1.22,2.34$ & 0.001 \\
\hline Q3 & 1.79 & $1.22,2.61$ & 0.003 & 1.36 & $0.96,1.93$ & 0.08 & 1.93 & $1.40,2.67$ & $<0.001$ \\
\hline Q4 & 1.67 & $1.22,2.45$ & 0.01 & 2.34 & $1.65,3.32$ & $<0.001$ & 3.28 & $2.33,4.61$ & $<0.001$ \\
\hline$p$ for trend & & & 0.01 & & & $<0.001$ & & & $<0.001$ \\
\hline \multicolumn{10}{|l|}{$\begin{array}{l}\text { Male subjects } \\
\quad(n=1677)\end{array}$} \\
\hline Q1 & 1 & & & 1 & & & 1 & & \\
\hline Q2 & 1.13 & $0.70,1.82$ & 0.61 & 0.91 & $0.58,1.43$ & 0.70 & 1.71 & $1.07,2.73$ & 0.03 \\
\hline$\widehat{Q} 3$ & 0.96 & $0.59,1.54$ & 0.85 & 1.00 & $0.64,1.55$ & 0.98 & 2.39 & $1.49,3.83$ & $<0.001$ \\
\hline Q4 & 1.10 & $0.68,1.78$ & 0.70 & 2.16 & $1.39,3.37$ & 0.001 & 2.92 & $1.78,4.78$ & $<0.001$ \\
\hline$p$ for trend & & & 0.09 & & & $<0.001$ & & & $<0.001$ \\
\hline \multicolumn{10}{|l|}{$\begin{array}{l}\text { Female subjects } \\
\quad(n=2771)\end{array}$} \\
\hline Q1 & 1 & & & 1 & & & 1 & & \\
\hline Q2 & 1.16 & $0.63,2.15$ & 0.63 & 1.02 & $0.60,1.76$ & 0.94 & 1.13 & $0.73,1.76$ & 0.58 \\
\hline$\hat{\mathrm{Q} 3}$ & 1.98 & $1.11,3.53$ & 0.02 & 1.48 & $0.89,2.46$ & 0.14 & 1.40 & $0.91,2.15$ & 0.13 \\
\hline Q4 & 2.11 & $1.17,3.79$ & 0.01 & 2.18 & $1.32,3.62$ & 0.003 & 2.59 & $1.66,4.05$ & $<0.001$ \\
\hline$p$ for trend & & & 0.003 & & & $<0.001$ & & & $<0.001$ \\
\hline
\end{tabular}

* The logistic model was adjusted for age, sex, BMI, GFR, hypertension, diabetes, dyslipidemia status, income level, smoking status, alcohol consumption, exercise status, use of vitamin supplements, and daily dietary intake of vitamin A. NAFLD, non-alcoholic fatty liver disease; AOR, adjusted odds ratio; CI, confidence interval; HSI, hepatic steatosis index; FSI, Framingham steatosis index; CNS, comprehensive NAFLD score; BMI, body mass index; GFR, glomerular filtration rate.

Table 4. AORs with 95\% CIs for association between $\alpha$-tocopherol and NAFLD assessed using different prediction models.

\begin{tabular}{|c|c|c|c|c|c|c|c|c|c|}
\hline \multirow{2}{*}{$\alpha$-Tocopherol } & \multicolumn{3}{|c|}{ NAFLD Assessed by HSI } & \multicolumn{3}{|c|}{ NAFLD Assessed by FSI } & \multicolumn{3}{|c|}{ NAFLD Assessed by CNS } \\
\hline & AOR * & $95 \% \mathrm{CI}$ & $p$-Value & AOR * & $95 \%$ CI & $p$-Value & AOR * & $95 \%$ CI & $p$-Value \\
\hline \multicolumn{10}{|l|}{$\begin{array}{l}\text { All subjects } \\
(n=4448)\end{array}$} \\
\hline Q1 & 1 & & & 1 & & & 1 & & \\
\hline Q2 & 1.14 & $0.80,1.63$ & 0.47 & 1.44 & $1.02,2.03$ & 0.04 & 1.37 & $0.99,1.88$ & 0.05 \\
\hline Q3 & 1.30 & $0.92,1.84$ & 0.14 & 2.08 & $1.49,2.91$ & $<0.001$ & 2.32 & $1.68,3.20$ & $<0.001$ \\
\hline$\widehat{\mathrm{Q}} 4$ & 1.44 & $1.01,2.05$ & 0.04 & 3.96 & $2.83,5.56$ & $<0.001$ & 3.39 & $2.44,4.71$ & $<0.001$ \\
\hline$p$ for trend & & & 0.03 & & & $<0.001$ & & & $<0.001$ \\
\hline \multicolumn{10}{|l|}{$\begin{array}{l}\text { Male subjects } \\
\quad(n=1677)\end{array}$} \\
\hline $\mathrm{Q} 1$ & 1 & & & 1 & & & 1 & & \\
\hline Q2 & 1.00 & $0.62,1.61$ & 0.99 & 1.58 & $1.00,2.50$ & 0.05 & 1.02 & $0.65,1.62$ & 0.92 \\
\hline Q3 & 1.41 & $0.89,2.23$ & 0.14 & 1.93 & $1.24,3.02$ & 0.004 & 1.76 & $1.10,2.81$ & 0.02 \\
\hline Q4 & 1.14 & $0.71,1.81$ & 0.59 & 4.34 & $2.76,6.84$ & $<0.001$ & 3.87 & $2.33,6.84$ & $<0.001$ \\
\hline$p$ for trend & & & 0.35 & & & $<0.001$ & & & $<0.001$ \\
\hline \multicolumn{10}{|l|}{$\begin{array}{l}\text { Female subjects } \\
\quad(n=2771)\end{array}$} \\
\hline Q1 & 1 & & & 1 & & & 1 & & \\
\hline$\widehat{\mathrm{Q}} 2$ & 1.38 & $0.76,2.50$ & 0.28 & 1.53 & $0.87,2.69$ & 0.14 & 1.71 & $1.08,2.70$ & 0.02 \\
\hline$\widehat{\mathrm{Q} 3}$ & 1.72 & $0.98,3.04$ & 0.06 & 2.10 & $1.24,3.53$ & 0.006 & 2.66 & $1.69,4.19$ & $<0.001$ \\
\hline Q4 & 2.02 & $1.14,3.59$ & 0.02 & 4.38 & $2.58,7.45$ & $<0.001$ & 3.39 & $2.16,5.32$ & $<0.001$ \\
\hline$p$ for trend & & & 0.01 & & & $<0.001$ & & & $<0.001$ \\
\hline
\end{tabular}

* The logistic model was adjusted for age, sex, BMI, GFR, hypertension, diabetes, dyslipidemia status, income level, smoking status, alcohol consumption, exercise status, and use of vitamin supplements. NAFLD, non-alcoholic fatty liver disease; AOR, adjusted odds ratio; CI, confidence interval; HSI, hepatic steatosis index; FSI, Framingham steatosis index; CNS, comprehensive NAFLD score; BMI, body mass index; GFR, glomerular filtration rate. 
We also performed an additional sensitivity analysis on the subjects including those excluded from the primary analysis because they had HBV, HCV, liver cirrhosis, and severe alcoholism. The results, which were consistent with the primary results regardless of gender, are summarized in Tables S2 and S3. Moreover, the results of the additional analysis for the association between $\alpha$-tocopherol with the cholesterol adjustment and NAFLD are presented in Table S4. As shown in the results, $\alpha$-tocopherol with the cholesterol adjustment was significantly associated with NAFLD except for HSI in all subjects and in male subjects.

\subsection{Subgroup Analysis According to Obesity and Diabetes}

Since obesity and diabetes are well-established risk factors for NAFLD, we analyzed the association between the vitamins and NAFLD after dividing the subgroups according to obesity and diabetes. The AORs and 95\% CIs for NAFLD comparing Q4 with Q1 for serum retinol and $\alpha$-tocopherol are summarized in Figure 3. According to the HSI prediction model, the association between the vitamins and NAFLD was either weak or non-significant, while the association remained significant for NAFLD according to FSI and CNS, except in diabetic patients according to FSI. The forest plot showed that NAFLD prevalence was positively associated with serum retinol and $\alpha$-tocopherol levels, especially in non-obese or diabetic subjects.

(a) serum retinol

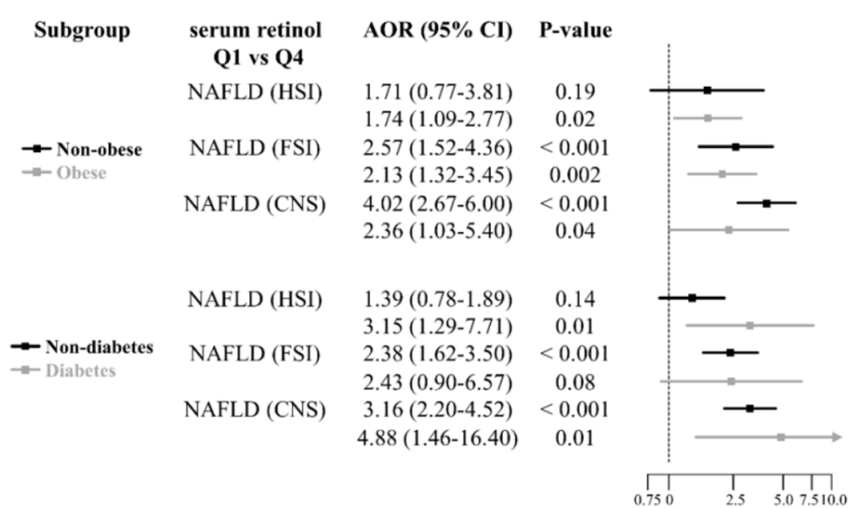

(b) $\alpha$-tocopherol

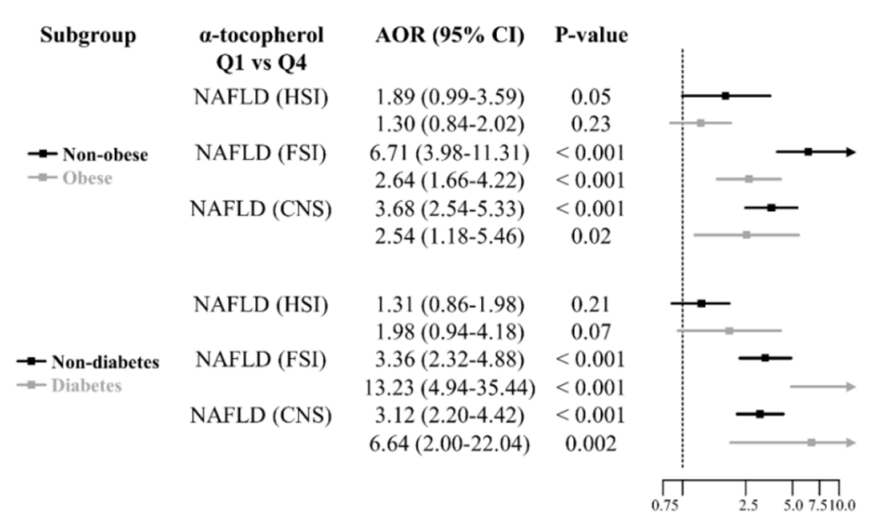

Figure 3. Subgroup analysis according to obesity and diabetes. The association between NAFLD status and vitamin (serum retinol and $\alpha$-tocopherol) levels was estimated using logistic regression models. The logistic model was adjusted for age, sex, BMI, GFR, hypertension, diabetes, dyslipidemia status, income level, smoking status, alcohol consumption, exercise status, use of vitamin supplements, and daily dietary intake of vitamin A. The association remained significant for NAFLD according to FSI and CNS, except in diabetic patients according to FSI. Q, quartile; AOR, adjusted odds ratio; $\mathrm{CI}$, confidence interval; NAFLD, non-alcoholic fatty liver disease; HSI, hepatic steatosis index; FSI, Framingham steatosis index; CNS, comprehensive NAFLD score. 


\subsection{Association between Vitamins and Advanced Fibrosis Assessed Using Different Prediction Models (BARD, FIB-4)}

When we examined the association between the vitamins and advanced fibrosis, we calculated the BARD and FIB-4 scores within the NAFLD population assessed by the three NAFLD prediction models. The proportions of patients with serum retinol levels according to the reference values for different advanced fibrosis scores are presented in Figure S4. The proportion of subjects with advanced fibrosis tended to decrease with increasing serum retinol levels in the analysis based on HSI and CNS, although the $p$-value for the trend was insignificant for FSI.

After serum retinol and $\alpha$-tocopherol levels were classified into quartile groups, we performed a multivariable-adjusted logistic regression analysis, the results of which are presented in Table 5. Most AORs and 95\% CIs for advanced fibrosis comparing Q1 for serum retinol to Q2, Q3, and Q4 were significant, although not within the NAFLD population assessed using the FSI model. Additionally, there was no significant association between $\alpha$-tocopherol and advanced fibrosis, except for the finding from the BARD model in FSI-based NAFLD subjects. Additional analyses with cholesterol-adjusted levels of $\alpha$-tocopherol did not show any significance for any of the associations (Table S5).

Table 5. AORs * with $95 \%$ CIs for association of serum retinol and $\alpha$-tocopherol with advanced fibrosis assessed using BARD and FIB-4.

\begin{tabular}{|c|c|c|c|c|c|c|c|c|c|c|c|c|c|c|c|c|c|c|}
\hline & \multicolumn{6}{|c|}{$\begin{array}{l}\text { NAFLD Assessed by HSI } \\
\qquad(n=862)\end{array}$} & \multicolumn{6}{|c|}{$\begin{array}{l}\text { NAFLD Assessed by FSI } \\
(n=1094)\end{array}$} & \multicolumn{6}{|c|}{$\begin{array}{l}\text { NAFLD Assessed by CNS } \\
\qquad(n=2093)\end{array}$} \\
\hline & \multicolumn{3}{|c|}{$\begin{array}{l}\text { BARD } \\
(n=416)\end{array}$} & \multicolumn{3}{|c|}{$\begin{array}{c}\text { FIB-4 } \\
(n=18)\end{array}$} & \multicolumn{3}{|c|}{$\begin{array}{l}\text { BARD } \\
(n=660)\end{array}$} & \multicolumn{3}{|c|}{$\begin{array}{c}\text { FIB-4 } \\
(n=40)\end{array}$} & \multicolumn{3}{|c|}{$\begin{array}{c}\text { BARD } \\
(n=1554)\end{array}$} & \multicolumn{3}{|c|}{$\begin{array}{c}\text { FIB-4 } \\
(n=64)\end{array}$} \\
\hline & AOR & $\begin{array}{l}95 \% \\
\text { CI }\end{array}$ & $\begin{array}{c}p- \\
\text { Value }\end{array}$ & AOR & $\begin{array}{l}95 \% \\
\text { CI }\end{array}$ & $\begin{array}{c}p- \\
\text { Value }\end{array}$ & AOR & $\begin{array}{c}95 \% \\
\text { CI }\end{array}$ & $\begin{array}{c}p- \\
\text { Value }\end{array}$ & AOR & $\begin{array}{l}95 \% \\
\text { CI }\end{array}$ & $\begin{array}{c}p- \\
\text { Value }\end{array}$ & AOR & $\begin{array}{c}95 \% \\
\text { CI }\end{array}$ & $\begin{array}{c}p- \\
\text { Value }\end{array}$ & AOR & $\begin{array}{c}95 \% \\
\text { CI }\end{array}$ & $\begin{array}{c}p- \\
\text { Value }\end{array}$ \\
\hline $\begin{array}{l}\text { Serum } \\
\text { retinol }\end{array}$ & & & & & & & & & & & & & & & & & & \\
\hline Q1 & 1 & \multirow{5}{*}{$\begin{array}{l}0.29 \\
0.80 \\
0.23 \\
0.65 \\
0.34 \\
0.97\end{array}$} & & 1 & \multirow{5}{*}{$\begin{array}{l}0.10 \\
1.90 \\
0.03, \\
1.18 \\
0.02, \\
0.83\end{array}$} & & 1 & \multirow{5}{*}{$\begin{array}{l}0.38 \\
0.89 \\
0.43 \\
1.04 \\
0.67 \\
1.67\end{array}$} & & 1 & \multirow{5}{*}{$\begin{array}{l}0.15 \\
1.05 \\
0.09 \\
0.83 \\
0.19 \\
1.25\end{array}$} & & 1 & \multirow{5}{*}{$\begin{array}{l}0.43 \\
0.82 \\
0.44 \\
0.86 \\
0.56 \\
1.13\end{array}$} & & 1 & \multirow{5}{*}{$\begin{array}{l}0.35 \\
1.34 \\
0.20 \\
0.92 \\
0.12 \\
0.67\end{array}$} & \\
\hline Q2 & 0.48 & & 0.01 & 0.43 & & 0.27 & 0.58 & & 0.01 & 0.40 & & 0.06 & 0.59 & & 0.002 & 0.68 & & 0.27 \\
\hline Q3 & 0.39 & & 0.001 & 0.18 & & 0.07 & 0.67 & & 0.07 & 0.28 & & 0.02 & 0.62 & & 0.004 & 0.43 & & 0.03 \\
\hline Q4 & 0.57 & & 0.04 & 0.12 & & 0.03 & 1.06 & & 0.82 & 0.49 & & 0.13 & 0.80 & & 0.21 & 0.29 & & 0.004 \\
\hline$p$ for trend & & & 0.04 & & & 0.02 & & & 0.57 & & & 0.08 & & & 0.39 & & & 0.002 \\
\hline \multicolumn{19}{|l|}{$\begin{array}{c}\alpha- \\
\text { tocopherol }\end{array}$} \\
\hline Q1 & 1 & \multirow{5}{*}{$\begin{array}{l}0.40 \\
1.07 \\
0.59 \\
1.63 \\
0.53 \\
1.46\end{array}$} & & 1 & & & 1 & \multirow{5}{*}{$\begin{array}{l}0.59 \\
1.36 \\
0.85 \\
2.01 \\
0.97 \\
2.35\end{array}$} & & 1 & \multirow{5}{*}{$\begin{array}{l}0.21 \\
1.35 \\
0.25 \\
1.66 \\
0.23 \\
1.66\end{array}$} & & 1 & \multirow{5}{*}{$\begin{array}{l}0.63 \\
1.16 \\
0.69 \\
1.28 \\
0.69 \\
1.31\end{array}$} & & 1 & \multirow{5}{*}{$\begin{array}{l}0.24 \\
1.08 \\
0.29 \\
1.21 \\
0.26 \\
1.19\end{array}$} & \\
\hline Q2 & 0.65 & & 0.09 & 0.85 & $\begin{array}{l}0.26 \\
2.77\end{array}$ & 0.99 & 0.89 & & 0.60 & 0.53 & & 0.18 & 0.86 & & 0.31 & 0.51 & & 0.08 \\
\hline Q3 & 0.98 & & 0.95 & 1.10 & $\begin{array}{l}0.27 \\
4.55\end{array}$ & 0.89 & 1.31 & & 0.23 & 0.65 & & 0.37 & 0.94 & & 0.68 & 0.59 & & 0.15 \\
\hline Q4 & 0.88 & & 0.61 & 0.46 & $\begin{array}{l}0.10 \\
2.25\end{array}$ & 0.34 & 1.51 & & 0.07 & 0.62 & & 0.34 & 0.95 & & 0.77 & 0.56 & & 0.13 \\
\hline$p$ for trend & & & 0.94 & & & 0.75 & & & 0.02 & & & 0.36 & & & 0.91 & & & 0.14 \\
\hline
\end{tabular}

* The logistic model was adjusted for age, sex, BMI, GFR, hypertension, diabetes, dyslipidemia status, income level, smoking status, alcohol consumption, exercise status, use of vitamin supplements, and daily dietary intake of vitamin A. NAFLD, non-alcoholic fatty liver disease; AOR, adjusted odds ratio; CI, confidence interval; HSI, hepatic steatosis index; FSI, Framingham steatosis index; CNS, comprehensive NAFLD score; BMI, body mass index; GFR, glomerular filtration rate.

\section{Discussion}

This study investigated the association between serum vitamin A (retinol) and E ( $\alpha$-tocopherol) levels and the prevalence of NAFLD in Korean adults. The prevalence of NAFLD was positively associated with serum retinol and $\alpha$-tocopherol levels independently from obesity and diabetes status. However, when patients with advanced fibrosis in the NAFLD group were evaluated, serum retinol levels and the presence of fibrosis tended to be inversely associated. Moreover, patients with serum retinol deficiency showed advanced fibrosis at a statistically higher frequency. Conversely, serum $\alpha$-tocopherol levels were not associated with the progression of advanced fibrosis.

There have been mixed reports on serum levels of vitamin A and E in NAFLD [20-22]. This could be due to study designs that included patients with all types of NAFLD, 
ranging from simple hepatic steatosis to NASH. Additionally, the prediction models used to estimate NAFLD varied in previous studies. Mazidi et al., demonstrating different results from ours, used only the fatty liver index (FLI) to define NAFLD [20]. In a validation study on the Korean population, FLI showed the lowest diagnostic performance compared to four other prediction models with an area under the receiver operating characteristic curve of 0.68 [12]. Therefore, the results from this study showing a negative association of serum vitamin A and E levels with NAFLD should be interpreted with caution. In our study, we used three different prediction models to measure NAFLD to increase the robustness of the study. On the other hand, a German study of 57 biopsy-proven NASH patients reported that, compared to healthy controls, the disease was significantly associated with lower serum levels of $\alpha$-tocopherol but not with lower serum levels of retinol [36]. The discrepancy between the prior study and ours may be due to the substantial differences in diagnostic criteria and the associated disease status.

Chen proposed a theory that a disturbance in vitamin A homeostasis in the liver may contribute to the development of NAFLD [37]. Specifically, he argued that in certain circumstances of the diseased state (i.e., changes in dietary or hormonal signals), hepatic stellate cells (HSCs) may lose their ability to store vitamin A in the liver [38]. Consequently, excessive vitamin A metabolism would occur, which could increase lipogenesis and contribute to fat accumulation in hepatocytes [39]. Additionally, the decrease in vitamin A storage in HSCs would induce more dietary vitamin A to be catabolized in the liver, resulting in the excessive release of retinol binding protein (RBP)/retinol complex [37]. This would act as a vicious cycle leading to a change in gene expression for glucose and lipid metabolism, eventually inducing NAFLD and NASH [40]. Based on our study results, it is speculated that, in the developmental stage and early phase of NAFLD, disturbed vitamin A homeostasis, which could affect lipid accumulation in the liver, results in elevated serum retinol levels due to an excessive release of RBP/retinol complex. As NAFLD progresses into advanced fibrosis, impaired vitamin A homeostasis causes severe vitamin A deficiency in the liver, resulting in a decrease in the serum retinol level [37]. This is consistent with a previous study investigating the association between vitamin A levels in the liver and NAFLD severity based on biopsy results: this study found that the more the hepatic vitamin A content decreased, the more NAFLD progressed [41]. However, this circumstance was not necessarily related to the plasma vitamin A level, given that serum retinol levels reflect hepatic vitamin A stores only when they are extremely deficient or excessive [42]. In sum, serum retinol deficiency in patients with NAFLD might be an indicator for fibrosis, although more data is needed to validate this association.

Only $1.1 \%$ of the subjects included in our study were deficient in vitamin E. As the serum $\alpha$-tocopherol level increased, the risk of developing NAFLD increased. This is consistent with the in vivo study by Bartolini et al. that showed that impaired cytochrome P-450-dependent vitamin E metabolism in the NAFLD-induced rat model resulted in the accumulation of $\alpha$-tocopherol in the liver [43]. Additionally, Nagita et al. found a correlation between hepatic and serum $\alpha$-tocopherol levels [44]. Therefore, it is conceivable that high serum $\alpha$-tocopherol levels were associated with NAFLD. In the subgroup analysis, serum $\alpha$-tocopherol levels showed a stronger association with NAFLD in non-obese or diabetic subjects, whereas there was no significant difference in serum $\alpha$-tocopherol levels between patients with simple steatosis without fibrosis and patients with advanced fibrosis, except for the finding from the BARD model in FSI-based NAFLD patients. These results have clinical implications because $\alpha$-tocopherol is used as a treatment for patients with NAFLD who have NASH, and some safety issues have been found [45].

NAFLD is a progressive disease that varies from simple hepatic steatosis to NASH. Our study evaluated the dynamic changes in serum vitamin A and E levels in patients with NAFLD according to the severity of the disease. According to our knowledge, it is the first study to assess differences in serum vitamin $\mathrm{A}$ and $\mathrm{E}$ levels in healthy people without NAFLD, patients with NAFLD but without advanced fibrosis, and patients with NAFLD with advanced fibrosis using nationally representative data. As to vitamin A, we found a 
correlation between serum retinol deficiency and advanced liver fibrosis. This finding is noteworthy in that it could provide a reference for determining when to conduct a biopsy to rule out NASH, although more studies are needed on this issue. Additionally, since vitamin $\mathrm{E}$ is used as a treatment for patients with NAFLD who have NASH, these results could provide clinicians references for NAFLD patient care at a micronutrient level.

Despite the strengths of our study, there are also several limitations. First, even though serum retinol levels decreased in patients with NAFLD who had advanced fibrosis, we could not confirm differences in serum retinol levels among the NASH subclasses according to histopathologic severity since there were no biopsy results for NAFLD. Second, information on supplementary vitamin $\mathrm{E}$ intake was not included in the data, unlike supplementary vitamin A intake. This could have introduced bias into our results. Third, we used noninvasive prediction models to assess NAFLD or advanced fibrosis rather than biopsy or imaging test results because those were unavailable due to the nature of the data source. Such scoring diagnoses may be less precise than biopsy-confirmed results or imaging tests. Nonetheless, to overcome this drawback, we used three and two different prediction models to diagnose NAFLD and advanced fibrosis, respectively, which was likely to improve the accuracy of the results: these models showed fairly reliable performance compared to each other and standard methods $[5,12,13]$. Further studies are needed to confirm our findings with populations based on the formal criteria for the diagnosis of the diseases.

\section{Conclusions}

In this study, we demonstrated that while circulating retinol and $\alpha$-tocopherol concentrations had a positive relationship with NAFLD prevalence, advanced fibrosis was only correlated with serum retinol levels. Therefore, in cases of serum retinol deficiency in patients with NAFLD, liver biopsy could be considered to rule out advanced fibrosis. Our findings could provide insight into NAFLD patient care at a micronutrient level.

Supplementary Materials: The following are available online at https:/ /www.mdpi.com/article/ 10.3390/nu13051720/s1, Figure S1: Proportion of subjects with serum retinol and $\alpha$-tocopherol levels according to quartile with predicted NAFLD prevalence (FSI) by quartile, Figure S2: Proportion of subjects with serum retinol and $\alpha$-tocopherol levels according to quartile with predicted NAFLD prevalence (CNS) by quartile, Figure S3: Proportion of subjects with serum retinol levels according to reference value with NAFLD scores, Figure S4: Proportion of subjects with serum retinol levels according to reference values for advanced fibrosis (assessed by BARD and FIB-4), Table S1: Prediction models for NAFLD and advanced fibrosis, Table S2: AORs with 95\% CIs for association of serum retinol with NAFLD assessed using different prediction models (all subjects including those with HBV, HCV, and liver cirrhosis and heavy alcoholics for the sensitivity analysis), Table S3: AORs with 95\% CIs for association of $\alpha$-tocopherol with NAFLD assessed using different prediction models (all subjects including those with $\mathrm{HBV}, \mathrm{HCV}$, and liver cirrhosis and heavy alcoholics for the sensitivity analysis), Table S4: AORs with $95 \%$ CIs for association between $\alpha$-tocopherol with cholesterol adjustment and NAFLD assessed using different prediction models, Table S5: AORs with 95\% CIs for association between $\alpha$-tocopherol with cholesterol adjustment and advanced fibrosis assessed using BARD and FIB-4.

Author Contributions: D.J., M.S. and J.S. were responsible for the conceptualization and design of the study; acquisition, analysis, and interpretation of the data; and drafting of the manuscript. M.S. performed the statistical analyses. All authors have read and agreed to the published version of the manuscript.

Funding: This study was supported by grants from the Basic Science Research Program through the National Research Foundation of Korea funded by the Ministry of Science and ICT (NRF2017R1E1A1 A01074298).

Institutional Review Board Statement: The study was conducted according to the guidelines of the Declaration of Helsinki and approved by the Institutional Review Board of Asan Medical Center (IRB number: 2020-1893). 
Informed Consent Statement: Informed consent was obtained from all subjects involved in the study.

Data Availability Statement: Data are available from the seventh Korea National Health and Nutrition Examination Survey (KNHANES VII, 2016-2018), conducted by the Korea Centers for Disease Control and Prevention (KCDCP). Data are freely available from KCDCP after signing up (https:/ / knhanes.cdc.go.kr, accessed on 14 December 2020).

Conflicts of Interest: The authors declare no conflict of interest.

\section{References}

1. Younossi, Z.M.; Koenig, A.B.; Abdelatif, D.; Fazel, Y.; Henry, L.; Wymer, M. Global epidemiology of nonalcoholic fatty liver disease-Meta-analytic assessment of prevalence, incidence, and outcomes. Hepatology 2016, 64, 73-84. [CrossRef] [PubMed]

2. European Association for the Study of the Liver; European Association for the Study of Diabetes; European Association for the Study of Obesity. EASL-EASD-EASO Clinical Practice Guidelines for the management of non-alcoholic fatty liver disease. J. Hepatol. 2016, 64, 1388-1402. [CrossRef] [PubMed]

3. Chalasani, N.; Younossi, Z.; Lavine, J.E.; Charlton, M.; Cusi, K.; Rinella, M.; Harrison, S.A.; Brunt, E.M.; Sanyal, A.J. The diagnosis and management of nonalcoholic fatty liver disease: Practice guidance from the American Association for the Study of Liver Diseases. Hepatology 2018, 67, 328-357. [CrossRef] [PubMed]

4. Long, M.T.; Pedley, A.; Colantonio, L.D.; Massaro, J.M.; Hoffmann, U.; Muntner, P.; Fox, C.S. Development and validation of the Framingham Steatosis Index to identify persons with hepatic steatosis. Clin. Gastroenterol. Hepatol. 2016, 14, 1172-1180.e2. [CrossRef]

5. $\quad$ Lee, Y.H.; Bang, H.; Park, Y.M.; Bae, J.C.; Lee, B.W.; Kang, E.S.; Cha, B.S.; Lee, H.C.; Balkau, B.; Lee, W.Y.; et al. Non-laboratorybased self-assessment screening score for non-alcoholic fatty liver disease: Development, validation and comparison with other scores. PLoS ONE 2014, 9, e107584. [CrossRef]

6. Torres, D.M.; Harrison, S.A. Diagnosis and therapy of nonalcoholic steatohepatitis. Gastroenterology 2008, 134, 1682-1698. [CrossRef]

7. Fierbinteanu-Braticevici, C.; Dina, I.; Petrisor, A.; Tribus, L.; Negreanu, L.; Carstoiu, C. Noninvasive investigations for non alcoholic fatty liver disease and liver fibrosis. World J. Gastroenterol. 2010, 16, 4784-4791. [CrossRef]

8. Yoneda, M.; Mawatari, H.; Fujita, K.; Iida, H.; Yonemitsu, K.; Kato, S.; Takahashi, H.; Kirikoshi, H.; Inamori, M.; Nozaki, Y.; et al. High-sensitivity C-reactive protein is an independent clinical feature of nonalcoholic steatohepatitis (NASH) and also of the severity of fibrosis in NASH. J. Gastroenterol. 2007, 42, 573-582. [CrossRef] [PubMed]

9. Kim, J.H.; Kwon, S.Y.; Lee, S.W.; Lee, C.H. Validation of fatty liver index and lipid accumulation product for predicting fatty liver in Korean population. Liver Int. 2011, 31, 1600-1601. [CrossRef]

10. Shen, Y.-N.; Yu, M.-X.; Gao, Q.; Li, Y.-Y.; Huang, J.-J.; Sun, C.-M.; Qiao, N.; Zhang, H.-X.; Wang, H.; Lu, Q.; et al. External validation of non-invasive prediction models for identifying ultrasonography-diagnosed fatty liver disease in a Chinese population. Medicine 2017, 96, e7610. [CrossRef]

11. Meffert, P.J.; Baumeister, S.E.; Lerch, M.M.; Mayerle, J.; Kratzer, W.; Völzke, H. Development, external validation, and comparative assessment of a new diagnostic score for hepatic steatosis. Am. J. Gastroenterol. 2014, 109, 1404-1414. [CrossRef] [PubMed]

12. Jung, T.Y.; Kim, M.S.; Hong, H.P.; Kang, K.A.; Jun, D.W. Comparative assessment and external validation of hepatic steatosis formulae in a community-based setting. J. Clin. Med. 2020, 9, 2851. [CrossRef] [PubMed]

13. Xiao, G.; Zhu, S.; Xiao, X.; Yan, L.; Yang, J.; Wu, G. Comparison of laboratory tests, ultrasound, or magnetic resonance elastography to detect fibrosis in patients with nonalcoholic fatty liver disease: A meta-analysis. Hepatology 2017, 66, 1486-1501. [CrossRef] [PubMed]

14. Harrison, S.A.; Oliver, D.; Arnold, H.L.; Gogia, S.; Neuschwander-Tetri, B.A. Development and validation of a simple NAFLD clinical scoring system for identifying patients without advanced disease. Gut 2008, 57, 1441-1447. [CrossRef]

15. Sterling, R.K.; Lissen, E.; Clumeck, N.; Sola, R.; Correa, M.C.; Montaner, J.; Sulkowski, M.S.; Torriani, F.J.; Dieterich, D.T.; Thomas, D.L.; et al. Development of a simple noninvasive index to predict significant fibrosis in patients with $\mathrm{HIV} / \mathrm{HCV}$ coinfection. Hepatology 2006, 43, 1317-1325. [CrossRef] [PubMed]

16. Pickett-Blakely, O.; Young, K.; Carr, R.M. Micronutrients in nonalcoholic fatty liver disease pathogenesis. Cell. Mol. Gastroenterol. Hepatol. 2018, 6, 451-462. [CrossRef]

17. Schiavo, L.; Busetto, L.; Cesaretti, M.; Zelber-Sagi, S.; Deutsch, L.; Iannelli, A. Nutritional issues in patients with obesity and cirrhosis. World J. Gastroenterol. 2018, 24, 3330-3346. [CrossRef]

18. Saeed, A.; Dullaart, R.P.F.; Schreuder, T.C.M.A.; Blokzijl, H.; Faber, K.N. Disturbed vitamin A metabolism in non-alcoholic fatty liver disease (NAFLD). Nutrients 2017, 10, 29. [CrossRef]

19. Podszun, M.C.; Chung, J.Y.; Ylaya, K.; Kleiner, D.E.; Hewitt, S.M.; Rotman, Y. 4-HNE immunohistochemistry and image analysis for detection of lipid peroxidation in human liver samples using vitamin E treatment in NAFLD as a proof of concept. J. Histochem. Cytochem. 2020, 68, 635-643. [CrossRef]

20. Mazidi, M.; Huybrechts, I.; Kengne, A.P. Associations between serum lipophilic antioxidants levels and non-alcoholic fatty liver disease are moderated by adiposity. Eur. J. Clin. Nutr. 2019, 73, 1088-1090. [CrossRef] 
21. Pettinelli, P.; Arendt, B.M.; Teterina, A.; McGilvray, I.; Comelli, E.M.; Fung, S.K.; Fischer, S.E.; Allard, J.P. Altered hepatic genes related to retinol metabolism and plasma retinol in patients with non-alcoholic fatty liver disease. PLoS ONE 2018, 13 , e0205747. [CrossRef] [PubMed]

22. Wallström, P.; Wirfält, E.; Lahmann, P.H.; Gullberg, B.; Janzon, L.; Berglund, G. Serum concentrations of $\beta$-carotene and $\alpha$ tocopherol are associated with diet, smoking, and general and central adiposity. Am. J. Clin. Nutr. 2001, 73, 777-785. [CrossRef] [PubMed]

23. Kim, T.; Kang, J. Association between serum retinol and $\alpha$-tocopherol levels and metabolic syndrome in korean general population: Analysis of population-based nationally representative data. Nutrients 2020, 12, 1689. [CrossRef] [PubMed]

24. Fan, J.G.; Kim, S.U.; Wong, V.W. New trends on obesity and NAFLD in Asia. J. Hepatol. 2017, 67, 862-873. [CrossRef]

25. Korea Centers for Disease Control and Prevention. The Korea National Health and Nutrition Examination Survey, 2019. KCDA Website. Available online: https:/ / knhanes.cdc.go.kr/ (accessed on 14 December 2020).

26. Kweon, S.; Kim, Y.; Jang, M.J.; Kim, Y.; Kim, K.; Choi, S.; Chun, C.; Khang, Y.H.; Oh, K. Data resource profile: The Korea National Health and Nutrition Examination Survey (KNHANES). Int. J. Epidemiol. 2014, 43, 69-77. [CrossRef]

27. Seo, M.H.; Lee, W.Y.; Kim, S.S.; Kang, J.H.; Kang, J.H.; Kim, K.K.; Kim, B.Y.; Kim, Y.H.; Kim, W.J.; Kim, E.M.; et al. 2018 Korean Society for the Study of Obesity guideline for the management of obesity in Korea. J. Obes. Metab. Syndr. 2019, 28, 40-45. [CrossRef]

28. Levey, A.S.; Bosch, J.P.; Lewis, J.B.; Greene, T.; Rogers, N.; Roth, D. A more accurate method to estimate glomerular filtration rate from serum creatinine: A new prediction equation. Modification of diet in renal disease study group. Ann. Intern. Med. 1999, 130, 461-470. [CrossRef]

29. Chobanian, A.V.; Bakris, G.L.; Black, H.R.; Cushman, W.C.; Green, L.A.; Izzo, J.; Joseph, L.; Jones, D.W.; Materson, B.J.; Oparil, S.; et al. The seventh report of the Joint National Committee on prevention, detection, evaluation, and treatment of high blood pressure: The JNC 7 report. JAMA 2003, 289, 2560-2571. [CrossRef]

30. Kim, M.K.; Ko, S.H.; Kim, B.Y.; Kang, E.S.; Noh, J.; Kim, S.K.; Park, S.O.; Hur, K.Y.; Chon, S.; Moon, M.K.; et al. 2019 Clinical practice guidelines for type 2 diabetes mellitus in Korea. Diabetes Metab. J. 2019, 43, 398-406. [CrossRef]

31. Committee for the Korean Guidelines for the Management of Dyslipidemia. 2015 Korean guidelines for the management of dyslipidemia: Executive summary (English Translation). Korean Circ. J. 2016, 46, 275-306. [CrossRef]

32. Lee, M.-R.; Jung, S.M.; Kim, H.S.; Kim, Y.B. Association of muscle strength with cardiovascular risk in Korean adults: Findings from the Korea National Health and Nutrition Examination Survey (KNHANES) VI to VII (2014-2016). Medicine 2018, 97, e13240. [CrossRef] [PubMed]

33. Kim, Y. Recommended intake and dietary intake of vitamin A for Koreans by unit of retinol activity equivalent. Korean J. Community Nutr. 2016, 21, 344-353. [CrossRef]

34. Lee, J.-H.; Kim, D.; Kim, H.J.; Lee, C.-H.; Yang, J.I.; Kim, W.; Kim, Y.J.; Yoon, J.-H.; Cho, S.-H.; Sung, M.-W.; et al. Hepatic steatosis index: A simple screening tool reflecting nonalcoholic fatty liver disease. Dig. Liver Dis. 2010, 42, 503-508. [CrossRef] [PubMed]

35. Ford, L.; Farr, J.; Morris, P.; Berg, J. The value of measuring serum cholesterol-adjusted vitamin E in routine practice. Ann. Clin. Biochem. 2006, 43, 130-134. [CrossRef] [PubMed]

36. Erhardt, A.; Stahl, W.; Sies, H.; Lirussi, F.; Donner, A.; Häussinger, D. Plasma levels of vitamin E and carotenoids are decreased in patients with Nonalcoholic Steatohepatitis (NASH). Eur. J. Med. Res. 2011, 16, 76-78. [CrossRef] [PubMed]

37. Chen, G. The link between hepatic vitamin A metabolism and nonalcoholic fatty liver disease. Curr. Drug Targets 2015, 16, 1281-1292. [CrossRef]

38. Li, D.; Friedman, S.L. Liver fibrogenesis and the role of hepatic stellate cells: New insights and prospects for therapy. $J$. Gastroenterol. Hepatol. 1999, 14, 618-633. [CrossRef]

39. Frey, S.K.; Vogel, S. Vitamin A metabolism and adipose tissue biology. Nutrients 2011, 3, 27. [CrossRef] [PubMed]

40. Zhang, Y.; Li, R.; Li, Y.; Chen, W.; Zhao, S.; Chen, G.J.B.; Biology, C. Vitamin A status affects obesity development and hepatic expression of key genes for fuel metabolism in Zucker fatty rats. Biochem. Cell Biol. 2012, 90, 548-557. [CrossRef]

41. Chaves, G.V.; Pereira, S.E.; Saboya, C.J.; Spitz, D.; Rodrigues, C.S.; Ramalho, A. Association between liver vitamin A reserves and severity of nonalcoholic fatty liver disease in the class III obese following bariatric surgery. Obes. Surg. 2014, 24, 219-224. [CrossRef]

42. Gibson, R.S. Principles of Nutritional Assessment; Oxford University Press: New York, NY, USA, 2005.

43. Bartolini, D.; Torquato, P.; Barola, C.; Russo, A.; Rychlicki, C.; Giusepponi, D.; Bellezza, G.; Sidoni, A.; Galarini, R.; Svegliati-Baroni, G.; et al. Nonalcoholic fatty liver disease impairs the cytochrome P-450-dependent metabolism of $\alpha$-tocopherol (vitamin E). J. Nutr. Biochem. 2017, 47, 120-131. [CrossRef] [PubMed]

44. Nagita, A.; Ando, M. Assessment of hepatic vitamin E status in adult patients with liver disease. Hepatology 1997, 26, 392-397. [CrossRef] [PubMed]

45. Klein, E.A.; Thompson, I.M., Jr.; Tangen, C.M.; Crowley, J.J.; Lucia, M.S.; Goodman, P.J.; Minasian, L.M.; Ford, L.G.; Parnes, H.L.; Gaziano, J.M.; et al. Vitamin E and the risk of prostate cancer: The selenium and vitamin E cancer prevention trial (SELECT). JAMA 2011, 306, 1549-1556. [CrossRef] [PubMed] 